\title{
Estimating Angles and Curvature Features In Grey Scale Images*
}

\author{
Zhimin Shao and Josef Kittler \\ Department of Electronic and Electrical Engineering \\ University of Surrey \\ Guildford, Surrey GU2 5XH \\ United Kingdom
}

\begin{abstract}
This paper presents a quantitative analysis of the error of the edge angle measurement obtained from edge detector in the presence of grey level gaussian noise. Our analysis shows that the uncertainty of the angle measurement is only related to the grey level noise power and the gradient of the edge point at which the angle is measured. Both image independent upper bound and image dependent estimate of the noise variance of the angle measurement are given.

With this result, we argue that one can provide better angle and curvature estimates by filtering the measurements adaptively according to the local uncertainty without having to compromise detail sensitivity and noise robustness. As an example of application, a new method of detecting curvature features is derived. The experimental results show that this algorithm works well, and in particular it can handle some difficult situations where other methods may fail.
\end{abstract}

\section{Introduction}

Contour curvature features are one of the most important information that can be used for object recognition from shape. The points of maximum convexity, concavity and inflexion partition the image curves into relative stable segments which then can be matched against each other. Sometime these points are also called critical or salient points. In classic mathematics, curvature is defined as the rate of the angle change along the arc length. Although some researchers argue that saliency in digitised image can be best captured by other means [5], curvature remains to be a generic concept of saliency on curves. The major challenge of curvature based approach is then how to extract the curvature feature points in the presence of noise accurately.

Generally there are two classes of approaches to the problem of restoring curvature information from digitised noisy image. An extensive comparison of curvature estimation methods has been reported by Worring [18].

The first class of methods is called orientation based. Accordingly, curvature estimates are obtained from angle information. Within this class of methods,

\footnotetext{
*This work was supported by ESPRIT BRA Project 7108, Vision as Process II
} 
there are two approaches. In the first approach, the angle information is obtained from a set of discrete edge pixel positions (e.g. [1],[14]). The other uses angles estimated from other sources such as the gradient information (e.g.[4] [2]). Obviously, if there is no gradient information available, e.g. in estimating curvature from binary images which is common in the literature on character recognition, we are constrained to use the first approach. A good comparative study of the methods that extract curvature from binary images can be found in [8].

The second class of methods named as path based do not estimate angles beforehand. Instead curvature is computed indirectly from a parametric approximation of the curve. The parametric model of the curve can be either global or local. The global curve fitting approach includes a number of spline fitting algorithms [12] [10] as well as the "snake" [7], and has the drawback of requiring an assumption of the form of the underlying contour [16]. Also the problem of optimal piecewise curve fitting often leads to a minimisation process that is so expensive that one has to settle for suboptimal or heuristic procedures. This is exemplified by the problem of optimal knot insertion in spline approximation of contours. The alternative is to use dynamic local models and treat the evolution of the local models as a filtering process [9] [11] [16]. In particular Sander's Kalman filtering method gives a good performance in terms of accuracy [16]. However such methods require an initial estimate of the appropriate scale of filtering which without knowing some statistical characteristics of the signal and the noise is quite difficult to give (see [16], the example of calculating the curvature of a logarithmic spiral). In general, path based methods perform poorly, with large bias on the angle and curvature estimation [18].

In this paper, we will present a new method of detecting curvature features. Our approach is orientation based since we will use the angle information calculated directly from the grey level gradient. Our method is different from the previous approaches in that we begin with a quantitative analysis of the noise present in the angle measurement, and then use the result of this analysis to guide the filtering and detection process. Our filter is adaptive because it can adjust itself according to the local noise condition. We derive our curvature feature detector using the optimal filter design advocated by Petrou as it guarantees optimal performance in terms of the objective criteria given by Spacek. Experimental results show that our curvature feature detection algorithm outperforms Gaussian smoothing method, and has the unique ability to adjust automatically according to the local SNR.

In section 2, we will describe some basic concepts and introduce the assumptions made about the noise in the signal. Section 3 presents a derivation of the power and the mean of noise on the angle calculated from the gradient information. Section 4 describes an adaptive filtering algorithm that uses results of Section 3 . Section 5 shows the experimental results and Section 6 draws some conclusions.

\section{Preliminaries}

Image restoration is concerned with the process of recovering measurement of the objects from noisy and imperfect image data. Image data acquired from a sensing-recording system usually suffers from both image dependent and image 
independent noise. Our ability of recovering original signal from noisy data depends on the extent of our knowledge about the degradation process [6, Chapter 8 , pp.267]. In this paper we specifically deal with the problem of recovering angle and curvature measurement from grey level images corrupted by image independent noise.

Most of edge detection processes involve thresholding of the gradient image. Edge pixels are characterised by local maxima of gradient magnitude values. Given an intensity image $f(x, y)$, the gradient image $g(x, y)$ is defined as

$$
g(x, y)=|\nabla f|=\sqrt{g_{x}^{2}+g_{y}^{2}}, \text { where } g_{x}=\frac{\partial f}{\partial x}, g_{y}=\frac{\partial f}{\partial y}
$$

Given that point $(x, y)$ is an edge pixel, its direction is define as :

$$
\theta\left(g_{x}, g_{y}\right)=\arctan \left(\frac{g_{y}}{g_{x}}\right)
$$

In practice, $g_{x}$ and $g_{y}$ are calculated by gradient masks. Here we are particularly interested in the class of separable masks since they introduce no correlations between the noise on $g_{x}$ and $g_{y}$. The simplest type of separable filter has a 1D form :

$$
H_{x}=\left[\begin{array}{lllll}
h_{x}^{0} & \cdots & 0 & \cdots & h_{x}^{N}
\end{array}\right], H_{y}=\left[\begin{array}{llllll}
h_{y}^{0} & \cdots & 0 & \cdots & h_{y}^{N}
\end{array}\right]^{T}
$$

Note that if the noise at all the points of image $f(x, y)$ is an Independently and Identically Distributed (IID) gaussian random variable, then the errors on the gradient estimates given by the $\mathrm{x}, \mathrm{y}$ masks are also gaussian. Thus we can assume the outputs of the gradient masks at a pixel $(x, y)$ to be

$$
g_{x}=g_{x 0}+\eta_{x}, \quad g_{y}=g_{y 0}+\eta_{y}
$$

where $g_{x 0}$ and $g_{y 0}$ are the true gradients and $\eta_{x}$ and $\eta_{y}$ are IID gaussian variables.

\section{Error in the Angle Measurement}

\subsection{Analysis of the error in calculating angles}

As Equation 2 suggests, the angle measurement can be treated as a two dimensional function of $\mathrm{x}, \mathrm{y}$ gradients. This functions is discontinuous at $g_{y}$-axis (where $\left.g_{x}=0\right)$ where $\arctan ()$ jumps from $-\pi / 2$ to $+\pi / 2$. Figure 3.1 shows the shape of $\arctan ()$ function.

Notice that, although the surface of $\theta\left(g_{x}, g_{y}\right)$ is discontinuous, it is continuously differentiable at $(-\infty, 0)$ and $(0,+\infty)$. Moreover,

$$
\lim _{g_{x} \rightarrow 0^{-}} \frac{\partial \theta}{\partial g_{x}}=\lim _{g_{x} \rightarrow 0^{+}} \frac{\partial \theta}{\partial g_{x}}=-\frac{1}{g_{y}}
$$

So generally when we exclude the origin $\left(g_{x}=0, g_{y}=0\right)$, partial derivatives $\frac{\partial \theta}{\partial g_{x}}$ and $\frac{\partial \theta}{\partial g_{y}}$ always exist and are finite. Hence we can apply Taylor expansion to 


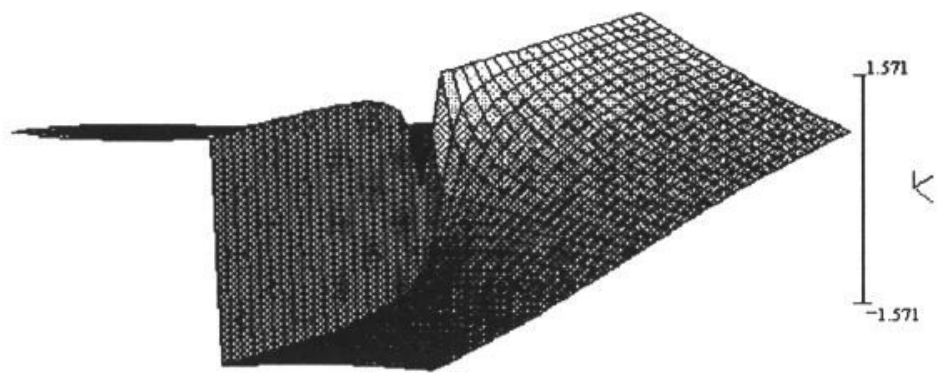

Figure 1: Surface $\theta\left(g_{x}, g_{y}\right)=\arctan \left(\frac{g_{y}}{g_{x}}\right)$

Equation 2 by substituting Equation 4 :

$$
\begin{aligned}
\theta\left(g_{x}, g_{y}\right)= & \theta\left(g_{x 0}+\eta_{x}, g_{y 0}+\eta_{y}\right) \\
= & \theta\left(g_{x 0}, g_{y 0}\right)+\left.\left(\frac{\partial \theta}{\partial g_{x}} \eta_{x}+\frac{\partial \theta}{\partial g_{y}} \eta_{y}\right)\right|_{\left(g_{x 0}, g_{y 0}\right)} \\
& +\left.\left(\frac{\partial}{\partial g_{x}} \eta_{x}+\frac{\partial}{\partial g_{y}} \eta_{y}\right)^{2} \theta\right|_{\left(g_{x 1}, g_{y 1}\right)}
\end{aligned}
$$

where $g_{x 1}$ and $g_{y 1}$ are bounded by $g_{x 0}, g_{x 0}+\eta_{x}$ and $g_{y 0}, g_{y 0}+\eta_{y}$. Define the error of angular measurement $\eta$ to be the 1st and 2nd order remainders:

$$
\eta\left(g_{x 0}, g_{y 0}\right)=\left.\left(\frac{\partial \theta}{\partial g_{x}} \eta_{x}+\frac{\partial \theta}{\partial g_{y}} \eta_{y}\right)\right|_{\left(g_{x 0}, g_{y 0}\right)}+\left.\left(\frac{\partial}{\partial g_{x}} \eta_{x}+\frac{\partial}{\partial g_{y}} \eta_{y}\right)^{2} \theta\right|_{\left(g_{x 1}, g_{y 1}\right)}
$$

Differentiating Equation 2 and knowing that $\eta_{x}$ and $\eta_{y}$ are IID gaussian variables, we can derive the mean and variance of the error of the angular measurement :

$$
\begin{gathered}
E\{\eta\}=0 \\
E\left\{\eta^{2}\right\}=\left(\frac{\sigma}{g_{0}}\right)^{2}+4\left(\frac{\sigma}{g_{1}}\right)^{4}
\end{gathered}
$$

where $g_{1}=\sqrt{g_{x 1}^{2}+g_{y 1}^{2}}$. Given that $g_{x 1}$ and $g_{y 1}$ are bounded within ranges $\left(g_{x 0}, g_{x 0}+\eta_{x}\right)$ and $\left(g_{y 0}, g_{y 0}+\eta_{y}\right)$, and $\frac{\sigma}{g_{x 0}}$ is small (typically less than $1 / 20$ for normal edge thresholding criteria), the variance of the error can be approximated by substituting $\frac{\sigma}{g_{x 1}} \approx \frac{\sigma}{g_{x 0}}$ :

$$
E\left\{\eta^{2}\right\} \approx\left(\frac{\sigma}{g_{0}}\right)^{2}+4\left(\frac{\sigma}{g_{0}}\right)^{4}
$$

Equation 8 and Equation 10 have several implications: 
1. Equation 8 shows that $\overline{\theta(x, y)}$ is an unbiased estimate of the angle at edge point $(x, y)$ if the error on $\mathrm{x}, \mathrm{y}$ gradient measurements are uncorrelated.

2. The mean square error (which somehow represents the uncertainty) of the angle measurement is only related to the signal to noise ratio of gradient measurement (which represents the "strength" of the edge). Given the estimate of the noise power in an image, we should be able to tell quantitatively about the uncertainty of our angle measurement at arbitrary point.

3. By utilising the uncertainty information, we can produce various kinds of adaptive filtering techniques to "restore" the angle signal from noisy data with generally higher accuracy than fixed scale filtering techniques. An example of combining optimal feature detector with this uncertainty information is given in the next section.

\section{Extracting Curvature Features}

\subsection{Our approach to the problem}

We aim to extract curvature from grey level images along contours defined by edge chains where gradient information is assumed to be available. We adopt the approach of filtering of edge angle measurement obtained from the gradient information as it offers the following advantages:

1. Angles estimated from edge pixel positions are more prone to error than those obtained directly from gradients. This is because the process of locating edge pixels often involves non-maxima suppression, thresholding, and ultimately a discretisation process. Furthermore, parametric models of the curves have to be fitted to the edge pixels to calculate the angles and this introduces additional approximation error. In Worring's paper [18], it has been shown that Duncan's method [4], which bases angle estimates on gradient with equidistant resampling is superior in performance to most of the path based methods.

2. In path based methods, nonlinear processes such as thresholding and discretisation of edge pixel positions make it difficult to relate the error of the estimated angle to grey level noise. Hence it is difficult to obtain the appropriate scale of smoothing to be applied to the raw data. In contrast as angles estimated from gradient information are not affected by any thresholding and discretisation process, it should be easier to analyse the propagation of the noise from grey level image to the angle estimate and study its effect analytically.

Asada and Brady [2] used a gaussian filter to smooth the orientation data to compute the curvature. Our method is similar to their approach but with two major differences:

1. Instead of using a gaussian filter, we use the criteria proposed by Spacek and the result from Petrou to obtain the optimal shape of the filter. This filter is 
optimised for good detection, localisation and suppression of false responses in detecting ramp shaped angle changes. An optimal filter for detecting inflexion points can be derived using the same procedure where the profile of angles at the inflexion point can be modelled by a gaussian function.

2. In section 3, we have shown how to estimate the noise of the angle measurement. The filter size $w$ in our method is calculated according to the local estimate of the noise, i.e. it can adjust itself adaptively.

\subsection{The optimal filter for ramp angle changes}

A curvature extremum is characterised by an abrupt angle change. Here we model the profile of this angle change with a ramp function:

$$
g(x)= \begin{cases}1-e^{-s x} / 2 & , x \geq 0 \\ e^{s x} / 2 & , \quad x \leq 0\end{cases}
$$

In principle the detection of curvature extrema follows the same rule that guides the design of edge detector (which is essentially the detection of gradient extrema in 2D space). Canny formulated three separate criteria for good extrema detection for 1D signal $g(x)$, namely the good detection criterion (maximum SNR):

$$
S=\frac{\left|\int_{-w}^{w} f(x) g(-x) d x\right|}{E\left(\eta^{2}\right) \sqrt{\int_{-w}^{w}|f(x)|^{2} d x}}
$$

localisation criterion :

$$
L=\frac{s^{2}\left|\int_{-w}^{w} f^{\prime}(x) g^{\prime}(-x) d x\right|}{E\left(\eta^{2}\right) \sqrt{\int_{-w}^{w}\left|f^{\prime}(x)\right|^{2} d x}}
$$

and suppression of false response:

$$
C=\frac{1}{w} \sqrt{\frac{\int_{-w}^{w}\left|f^{\prime}(x)\right|^{2} d x}{\sqrt{\int_{-w}^{w}\left|f^{\prime \prime}(x)\right|^{2} d x}}}
$$

Spacek combined criteria $12-14$ into a single criterion function $P$

$$
P=\frac{s^{4}\left|\int_{-w}^{0} f(x)\left(1-e^{s x}\right) d x \int_{-w}^{0} f(x) e^{s x} d x\right|^{2}}{w^{2} \int_{-w}^{0}|f(x)|^{2} d x \int_{-w}^{0}\left|f^{\prime \prime}(x)\right|^{2} d x}
$$

and applied to it to the problem of detecting a step signal. Petrou [13] extended his work to ramp signals of the form given in Equation 11 to find the following filter:

$$
\begin{aligned}
f(x) & =e^{A x}\left[L_{1} \sin (A x)+L_{2} \cos (A x)\right] \\
& +e^{-A x}\left[L_{3} \sin (A x)+L_{4} \cos (A x)\right]+L_{5} x+L_{6} e^{s x}+L_{7}
\end{aligned}
$$

where $L_{1} \cdots L_{7}$ have been optimised numerically for different filter size $w$ [13]. This is the generic form of the filter we have adopted for detecting curvature extrema in our work. 


\subsection{Dynamic determination of the filter size $w$}

Many researchers have noted the problem of choosing an optimal filter size $w$ to simultaneously maximise both SNR and localisation criteria (Equation 12 and 13) [3] [13]. A large filter size normally gives a better signal to noise ratio, small filter size can improve the accuracy on locating the peak. In the problem of edge detection, Canny argued that since the signal-to-noise ratio of each edge is likely to be different in an image, the size of the operator must be changed dynamically by the algorithm and this requires a local estimate of the noise energy in the region surrounding the candidate edge[3]. Similarly, in the problem of detecting curvature extrema we have shown that the noise power of the angle measurement is a point-dependent function (Equation 10), hence we should make our filter size adjustable according to the local angular noise estimate given by Equation 10 .

It is reasonable to assume that in many images the strength of edge is likely to be constant along a curve for some length, and thus within a certain region of support the angular noise is stationary. So for each edge segment where edge pixels have similar gradient, we may begin searching for a filter size $w$ by assuming $E\left(\eta^{2}\right) \equiv \overline{E\left(\eta^{2}\right)} . \overline{E\left(\eta^{2}\right)}$ is the average of m.s. angular errors estimated by Equation 10.

When $E\left(\eta^{2}\right)$ is given, in principle we can solve Equation 12 for the value of $w$ so that the detection criterion $S$ (which is the signal-to-noise ratio) equals a given constant. By fixing the value of $S$, the value of objective function $L$ and $C$ are also fixed, and we have a constrained optimality where for a given demand of signal-to-noise ratio, we obtain a locally optimal curvature feature detector. On the other hand, one may want to find an appropriate $w$ from Equation 13 to satisfy a specification of localisation. In either case, the $w$ found might not be a global optimum. Note that if we attempt to optimise Equation 15 for $w$ directly, we will find that the larger $w$ is the greater $P$ is. This is because the larger the filter the more the edge "looks" to the filter like an ideal step edge [13].Obviously, a large smoothing kernel is not always desirable, as it may perform poorly in terms of localisation and also cause feature interference.

In practice, both detection and localisation criteria (Equation 12 and Equation 13) when treated as functions of $w$ are generally monotonic. We can therefore solve either one of the two equations for $w$ using logarithmic search. As the number of permissible values of $w$ is rather limited, this search can be quite fast (normally less than 10 iterations to converge).

Now we summaries our curvature feature detection algorithm:

1. Perform separable optimal filtering based edge detection. Record angle and gradient information and the estimate of grey level noise.

2. Split edge strings into segments so that in each segment the sum of the variance of the gradient is less than or equal to a given constant $G$. Within each segment, the filter size will be constant.

3. For each segment,

- Perform logarithmic search for the optimal filter size $w$ which satisfies either a given value of $S$ in Equation 12 or of $L$ in Equation 13 


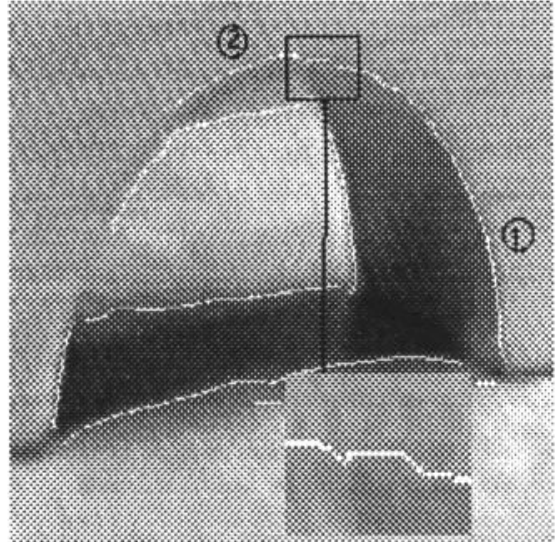

(a) The two segments 1 and 2 of the edge of the inner arch have different error variance when the strength changes

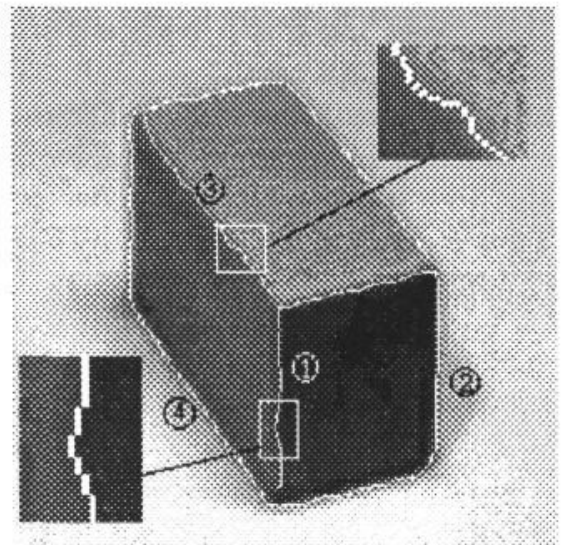

(b) Different edges of a box have different errors of angle estimate due to different strength: edge 1 and edge 2 (and similarly edge 3 and 4 ) have same angle but different error variance.

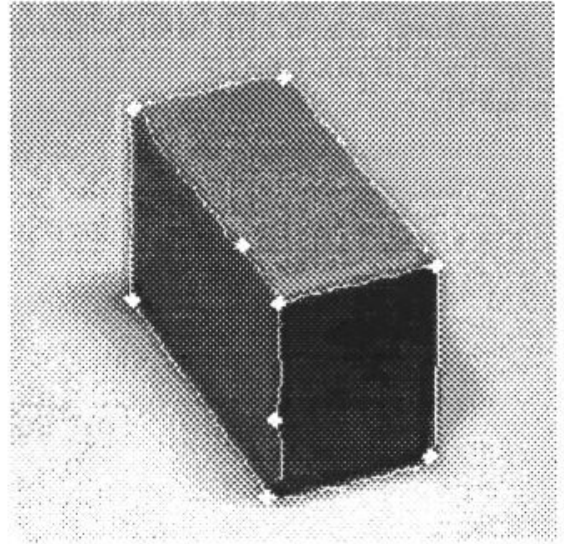

(c) Corners of the box in (b) detected by the fixed width filter $(w=6)$

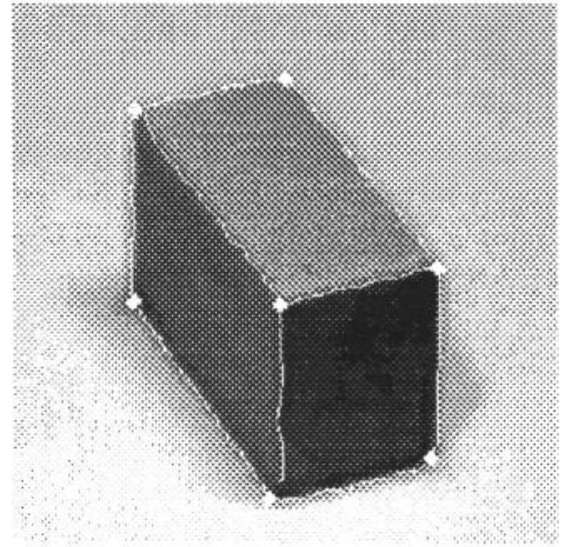

(d) Corner of the box in (b) detected by the adaptive curvature feature detector

Figure 2: Change of edge strength causes change of error variance : locations on the edges where a false maxima are likely to be detected by the fixed size filter are shown in the enlarged boxes. 
- Select filter weights from filter table, and generate filter mask. Convolve the mask with angle data.

- Differentiate the filter output and threshold to get curvature extrema.

4. Perform extrema selection by ranking and non-maxima suppression.

\section{Experimental Results}

Figures 2(a) and 2(b) show two test images. Note that in Figure 2(a), the marked edge becomes more noisy when the gradient changes from segment 1 to segment 2. This justifies our error analysis results (Equation 8, Equation 10.) From Equation 12 we know that as the edge becomes more noisy, larger filter kernel is required to maintain the desired SNR criterion at output of the filter. Figure 2(c) demonstrates the corners detected by a fixed size smoothing kernel. We can see that due to the change in the angular SNR, two false maxima were detected. By contrast in Figure 2(d), these false maxima are suppressed by our adaptive filter as the kernel size increased automatically when the noisy edges were encountered.

\section{Remarks and Conclusion}

In this paper we have presented an analysis of the error of angle information provided by the edge detector. We showed that if the errors in $x, y$ gradient are uncorrelated, the error of the angle measurement at any edge point is only related to the local SNR of the gradient 10 . However, we are not entirely constrained to the class separable edge detectors. For an arbitrary edge detection mask, we are able to tell quantitatively the amount of correlation introduced in the $x, y$ gradient. By similar analysis, using the means, variances of the gradient components and the correlation between them, we can derive formulae for mean and variance of the error of the angle measurement. In this case, the mean can be non-zero for asymmetric masks, which means a noise cancelling is required before applying the filter.

The optimal filter formula 16 is a one dimensional application of the edge detection theory proposed by Canny and Spacek and extended by Petrou. In fact, the problems of edge detection and curvature feature detection from angle data are subject to the same principles and measure of quality which can be viewed as a general theory of feature detection.

\section{References}

[1] I.M.Anderson, J.C.Bezdek. Curvature and Tangential Deflection of Discrete Arcs : A Theory Based on Commutator of Scatter Matrix Pairs and Its Application to Vertex Detectioon in Planar Shaped Data. IEEE PAMI-6, pp.27, Jan. 1984. 
[2] H.Asada, M.Brady. The Curvature Primal Sketch. IEEE PAMI-8, pp.131, Jan. 1986.

[3] J. Canny. A Computational Approach to Edge Detection. IEEE PAMI-8, pp.679, Jan. 1986.

[4] J.S.Duncan, A.W.M.Scmeulders, F.Lee, B.L.Zaret. Measurement of End Diastolic Shape Deformity Using Bending Energy. Computer in Cardiology, IEEE Press, 1988.

[5] M.A.Fischler, H.Wolf. Locating Perceptually Salient Points on Planar Curves. IEEE PAMI-16, No.2, pp.131, Feb. 1994.

[6] A.K.Jain. Fundamentals of Digital Image Processing. Prentice-Hall, 1989.

[7] M. Kass, A. Witkin, and D. Terzopoulos Snakes: active contour models. IJCV 1, pp.321-331, 1988

[8] R.Legault, C.Y.Suen. A Comparison of Methods of Extracting Curvature Features. IAPR Vol.3, pp.134, 1992.

[9] D.G.Lowe Organisation of Smooth Image Curves at Multiple Scales. ICCV, pp.558, Tampa, 1988.

[10] G.Medioni, Y.Yasumoto. Corner Detection and Curve Representation Using B-Splines. CGVIP, 1987.

[11] F.Mokhtarian, A.Macworth. Scale-based Description and Recognition of Planar Curves and Two-dimentional Shapes. IEEE PAMI-8, pp.34, Jan.1986.

[12] T.Pavlidis. Application of Splines to Shape Description. Visual Forms Analysis and Recognition, Proceedings of International Workshope on Visual Form, pp.431, May. 1991.

[13] M.Petrou, J.Kittler. Optimal Edge Detectors for Ramp Edges. IEEE PAMI13, pp.483, May.1991.

[14] A.Rosenfeld, E.Johnston. Angle Detection in Digital Curves. IEEE Transactions on Computing, Vol.C-24, pp.940, 1973.

[15] L.A.Spacek. Edge Dectection and Motion Detections. Image Vision Computing, Vol.4, 1986.

[16] P.T.Sander. Estimating Curvature by Kalman Filters. Visual Forms - Analysis and Recognition, Proceedings of International Workshope on Visual Form, pp.469, May. 1991.

[17] B.Widrow, S.D.Stearns. Adaptive Signal Processing. Prentice-Hall Signal Processing Series, Prentice-Hall 1992.

[18] M.Worring, A.W.M.Smeulders. The Accuracy and Precision of Curvature Estimation Methods. IAPR, Vol.3, pp.139, 1992. 